\title{
Autobiographical memory in non-amnesic alcohol-dependent patients
}

\author{
ARNAUD D’ARGEMBEAU ${ }^{1 *}$, MARTIAL VAN DER LINDEN ${ }^{1,2}$, \\ PAUL VERBANCK ${ }^{3}$ AND XAVIER NOËL ${ }^{3}$ \\ ${ }^{1}$ Cognitive Psychopathology Unit, University of Liège, Belgium; ${ }^{2}$ Cognitive Psychopathology and \\ Neuropsychology Unit, University of Geneva, Switzerland; ${ }^{3}$ Addiction Clinic, Department of Psychiatry, \\ Brugmann University Hospital, Free University of Brussels, Belgium
}

\begin{abstract}
Background. Chronic alcohol abuse is associated with a wide range of cognitive deficits. However, little is known about memory for real-life events (autobiographical memory) in non-amnesic alcoholic patients. The purpose of this study was to investigate $(a)$ non-amnesic alcoholics' ability to recall specific autobiographical memories and $(b)$ their subjective experience when they access specific memories.

Method. Twenty non-amnesic (without Korsakoff syndrome) recently detoxified alcoholics and 20 healthy controls completed the Autobiographical Memory Test (AMT), which assesses the frequency of specific (versus general) memories recalled in response to cue words, and the Memory Characteristics Questionnaire (MCQ), which assesses subjective experience (e.g. the amount of sensory and contextual details experienced) when remembering specific events.
\end{abstract}

Results. Alcoholic patients recalled specific memories less frequently and general memories more frequently than healthy controls. Nevertheless, when a specific past event was accessed, alcoholic patients subjectively experienced as many sensory and contextual details as controls.

Conclusions. These findings suggest that non-amnesic alcoholics have difficulties strategically accessing event-specific autobiographical knowledge, which might result from changes in frontal lobe function that are associated with alcoholism.

\section{INTRODUCTION}

Chronic alcohol abuse is associated with a wide range of cognitive deficits (Parsons, 1998; Moselhy et al. 2001; Scheurich, 2005). Alcoholdependent patients perform poorly on tasks assessing working memory and executive functions, including planning ability, inhibition, rule detection, and coordination of dual tasks (Sullivan et al. 2000; Noël et al. 2001). Impairments are also observed in tests of long-term memory, for both verbal and non-verbal stimuli (Grant, 1987), in learning novel associations (Brandt et al. 1983), and in remembering the

\footnotetext{
* Address for correspondence: Arnaud D’Argembeau, Cognitive Psychopathology Unit, University of Liège, Boulevard du Rectorat, 3 (B33), 4000 Liège, Belgium.

(Email: a.dargembeau@ulg.ac.be)
}

source of recently presented information (Schwartz et al. 2002). These findings suggest that alcoholics find it difficult to recollect details about their past experiences. However, the memory tasks used in most studies (memory for lists of words or pictures) differ from memory for real-life events (autobiographical memory) in important aspects, such as their time-frame and their significance in terms of personal goals and emotional salience (Conway, 2001; Gilboa, 2004). Therefore, the recollection of real-life events by alcohol-dependent patients should be considered in its own right.

Although it is well established that alcoholic patients with Korsakoff syndrome show a severe retrograde amnesia (Kopelman \& Kapur, 2001), relatively little is known about autobiographical 
memory (AM) in non-amnesic alcoholic patients. Some studies have used self-report questionnaires to assess the frequency of everyday memory lapses (e.g. 'telling someone a story or joke that you have told them before') and prospective memory difficulties (e.g. 'I forgot to pass on a message to someone'), and found that heavy alcohol users report more impairments compared to low or non-users of alcohol (Heffernan et al. 2002; Ling et al. 2003). Another study examined the temporal distribution of AMs recalled in response to cue words, showing that recently detoxified alcoholics recalled fewer memories from their recent past and more memories from their early adulthood than healthy controls (Fitzgerald \& Shifley-Grove, 1999). These studies suggest that alcoholism is associated with some AM impairments. However, the precise AM components that are affected and the mechanisms that underlie these impairments remain to be investigated.

In this perspective, it may prove fruitful to consider a recent and well-supported model of AM (Conway \& Pleydell-Pearce, 2000; Conway, 2001). This model conceives of AMs as transitory mental representations that are constructed from an autobiographical knowledge base that contains knowledge at different levels of abstraction. Lifetime periods are the most abstract knowledge structures, containing knowledge common to a delineated period of time that is defined by a theme (e.g. ' when I was at high school'). General events are more specific than lifetime periods and contain information related to repeated events (e.g. 'Sundays at Grandma's place') or events extended in time (e.g. 'my holiday in France'). The third level of autobiographical knowledge contains experience-near sensory-perceptual knowledge (i.e. images, feelings) concerning specific events that happened at a particular time and place. The recall of $\mathrm{AMs}$ is often intentional and effortful. It is a complex process that typically begins with the search for a general event, which is then used as a cue to access sensoryperceptual details concerning a specific event. When accessed, this event-specific knowledge enables one to 'see' in one's mind the location where the remembered event took place and the persons who were present, to feel what one felt, to remember what one thought, and so forth
(Johnson et al. 1988; Rubin et al. 2003). Typically, then, an AM consists of a general event plus event-specific knowledge. However, in some cases, autobiographical knowledge is accessed independently of event-specific information, resulting in the retrieval of more abstract autobiographical information (e.g. knowledge about repeated events) (Williams, 1996; Van Vreeswijk \& De Wilde, 2004).

Drawing on this model of AM, the first aim of this study was to examine whether non-amnesic alcoholics differed from healthy controls in terms of the frequency with which they retrieve specific versus general (repeated or extended) AMs in response to cue words. This was assessed by means of the Autobiographical Memory Test (AMT; Williams \& Broadbent, 1986), which has been used extensively to investigate AM specificity in various clinical populations, including depression (Van Vreeswijk \& De Wilde, 2004; Williams, 1996), post-traumatic stress disorder (McNally et al. 1995) and schizophrenia (Riutort et al. 2003). As the retrieval of specific AMs is time-consuming and depends crucially on sufficient executive resource capacity (Conway \& Pleydell-Pearce, 2000), which has been shown to be reduced in alcoholism (Sullivan et al. 2000; Noël et al. 2001), it is predicted that alcoholics will retrieve specific memories less frequently and general memories more frequently than healthy controls.

Our second aim was to investigate the conscious experience of alcoholic patients when they access specific AMs. This was done by requiring participants to retrieve specific events and, keeping these specific events in mind, to rate the phenomenal characteristics of their memories (e.g. the amount of sensory and contextual details they subjectively experience) with the Memory Characteristics Questionnaire (MCQ; Johnson et al. 1988; D'Argembeau \& Van der Linden, 2004). In addition, as recent findings suggest that the ability to remember the past and the ability to imagine the future are intimately related (Suddendorf \& Corballis, 1997; Atance \& O'Neill, 2001; D'Argembeau \& Van der Linden, 2004), we also explored (by means of MCQ ratings) whether alcoholics differ from healthy controls in their conscious experience when they project themselves into specific future events. 
Table 1. Demographic and clinical variables for alcoholic patients and healthy controls

\begin{tabular}{lccc}
\hline \hline Parameter & Alcoholics $(n=20)$ & Controls $(n=20)$ & $t(38)$ \\
\hline Age (years) & $44 \cdot 7 \pm 8 \cdot 4$ & $44 \cdot 6 \pm 8 \cdot 8$ & $0 \cdot 04$ \\
Gender (M/F) & $17 / 3$ & $17 / 3$ & - \\
Education (years) & $12 \cdot 5 \pm 3 \cdot 4$ & $13 \cdot 4 \pm 3 \cdot 2$ & $-0 \cdot 82$ \\
Daily alcohol consumption (g ethanol) & $244 \cdot 5 \pm 83 \cdot 3$ & $13 \cdot 6 \pm 15 \cdot 3$ & $12 \cdot 19 * *$ \\
Number of prior detoxification & $3 \cdot 6 \pm 2 \cdot 0$ & - & - \\
Abstinence (days) & $19 \cdot 5 \pm 2 \cdot 6$ & $3 \cdot 6 \pm 2 \cdot 3$ & $4 \cdot 79^{* *}$ \\
BDI & $13 \cdot 6 \pm 9 \cdot 1$ & $37 \cdot 8 \pm 8 \cdot 6$ & $5 \cdot 04^{* *}$ \\
STAI trait & $56 \cdot 3 \pm 14 \cdot 0$ & $35 \cdot 2 \pm 10 \cdot 3$ & $2 \cdot 49^{*}$ \\
STAI state & $45 \cdot 2 \pm 14 \cdot 6$ & & \\
\hline \hline
\end{tabular}

BDI, Beck Depression Inventory - Short Form; STAI, State Trait Anxiety Inventory.

$* p<0 \cdot 05, * * p<0 \cdot 001$.

\section{METHOD}

\section{Participants}

Twenty alcohol-dependent patients were recruited from the Alcohol Detoxification Program of the Psychiatric Institute, Brugmann University Hospital, Brussels, Belgium. They all underwent complete medical, neurological and psychiatric examinations at the time of the selection. The participants had to meet DSM-IV criteria for alcohol dependency (as assessed by a board-certified psychiatrist). Reasons for exclusion were other current DSM-IV Axis I diagnoses, Korsakoff syndrome, a history of significant medical illness, head injury resulting in a loss of consciousness for longer than 30 minutes that would have affected the central nervous system, use of other psychotropic drugs or substances that influence cognition, and overt cognitive dysfunction. To increase the reliability of information, alcoholic patients and their families were interviewed separately. Blood levels of folate, vitamin $\mathrm{B} 12$ and $\beta$-carotene were measured. The detoxification regimen consisted of the administration of B vitamins, and a gradual decrease in doses of sedatives (diazepam). Current clinical status was rated using the Beck Depression Inventory short form (BDI; Beck et al. 1974) and the Spielberger State Trait Anxiety Inventory (STAI trait \& STAI state; Spielberger, 1993). All the patients were tested between 16 and 23 days after they stopped drinking, and at least 5 days after administration of diazepam had been stopped. The demographic data are shown in Table 1.

Twenty healthy controls matched for sex, age and educational level to the alcoholic patients were recruited by word of mouth from the community. We excluded individuals who met an Axis I psychiatric diagnosis, as assessed by the Structural Clinical Interview for DSM-IV, those who had been diagnosed with a drug abuse disorder during the year preceding their enrolment in the study, or who had consumed more than $54 \mathrm{~g} /$ day of alcohol for longer than a month. On the basis of their medical history and physical examination, they were judged to be medically healthy. Control participants were asked to avoid the use of drugs, including narcotic pain medication, for the 5 days prior to testing, and to avoid alcohol consumption during the 24 hours prior to testing. After a complete description of the study, written informed consent was obtained from all the participants.

\section{Autobiographical memory assessment}

\section{AMT (Williams \& Broadbent, 1986)}

Participants were asked to recall events that had happened to them in the past in response to 10 emotional cue words (five positive, five negative). It was emphasized that the events recalled should be specific (events that lasted less than 1 day); some examples were provided to illustrate what would or would not be considered as a specific event. In addition, the events had to have occurred at least 1 year ago. This was because we observed in a pilot study (with other participants) that recently detoxified alcoholics almost exclusively recalled events they had just experienced during the detoxification program when the retrieval period was not specified. The cues were written on cards that 
were presented one by one, alternating positive and negative words. Participants were given 60 seconds to retrieve a specific memory in response to each cue; if their first response was not a specific memory, they were prompted again to retrieve a specific memory (e.g. 'Can you think of a specific episode?'). All responses were tape-recorded and then transcribed for scoring.

Participants' responses to each cue word were analyzed by two independent raters. When participants did not respond or when they gave a response that was not a memory (e.g. 'You know I am the kind of person who suppresses my anger' in response to the cue word 'angry'), this was scored as an omission. The remaining memory responses were then categorized as one of three types (Williams et al. 2000): specific (memory for an event that happened at a particular time and place and did not last longer than a day, e.g. 'going to a concert last summer'), extended (memory of a particular time-period lasting longer than a day, e.g. 'my three-week holiday in France'), and categoric (a memory response that summarized a number or category of events, e.g. 'going to concerts as a child'). Agreement between the two raters was very good $(\kappa=0.83)$; discrepancies were resolved by discussion.

\section{Phenomenal characteristics associated with projecting oneself into past and future events (D'Argembeau \& Van der Linden, 2004)}

Participants were asked to remember two events (one positive, one negative) that they had personally experienced in the past and to imagine two events (one positive, one negative) that might happen to them in the future. Detailed instructions explained that the events participants were to recall or imagine had to be precise and specific; for future events it was also mentioned that the events had to be things that might reasonably happen to them in the future. To prevent alcohol-dependent patients from recalling events they had just experienced during the detoxification program, all the participants were required to recall events that occurred at least 1 year ago. Similarly, they were asked to imagine future events that might happen to them in 1 year or more. Once a specific event had been selected, participants first wrote a brief description of the event, and then closed their eyes and tried to remember/imagine the event in as much detail as possible (i.e. remembering/ imagining the setting and course of the event, the persons and objects that were/would be present, and so forth) in order to mentally 'reexperience' (or 'pre-experience') it.

Immediately after having remembered/ imagined each event, participants rated their conscious experience with seven-point rating scales adapted from the MCQ (Johnson et al. 1988). An index of sensory details was computed by averaging responses to three items: amount of visual details, amount of sounds, and amount of smell/taste $(1=$ none, $7=\mathrm{a}$ lot $)$. Representation of contextual information was assessed with four items: clarity of location, clarity of the spatial arrangement of objects, clarity of the spatial arrangement of people, and clarity of the time of day $(1=$ not at all clear, $7=$ very clear). Self-referential information was assessed with three items: representation of one's own behavior, representation of what one said/would say, and representation of what one thought/would think $(1=$ none, $7=$ very detailed). Other-referential information was assessed with two items: representation of the behavior of other people and representation of what other people said/would say $(1=$ none, $7=$ very detailed). Participants were also asked to report the visual perspective they took in their memory (Nigro \& Neisser, 1983), depending on whether they 'saw' themselves in their memory (observer perspective) or saw the scene from their own perspective (field perspective; from $-3=$ entirely looking through my eyes to $+3=$ entirely observing myself from an outside point of view). Finally, participants rated each event for positive emotion $(1=$ no positive emotion, $7=$ very intense positive emotion) and negative emotion $(1=$ no negative emotion, $7=$ very intense negative emotion), and they estimated its temporal distance from the present (in months).

\section{Procedure}

Participants were tested individually in two sessions. The phenomenal characteristics associated with projecting oneself into past and future events were assessed during the first session, and the AMT was completed during the second session. The phenomenal characteristics task was always administered in the first session, so 
that participants had the opportunity to retrieve specific memories (and were therefore able to clearly understand what a specific memory is) before being assessed with the AMT. The two testing sessions were separated by one or two day(s) to minimize the possibility that the phenomenal characteristics task influenced AMT performance.

\section{Data analysis}

With regard to the AMT, initial 2 (group) by 2 (valence of the cues) analyses of variance (ANOVAs) indicated that the valence of the cues did not have any significant effect and did not interact with the group. Therefore, for the sake of brevity, data were collapsed across positive and negative cues, and between-group differences were examined with $t$ tests. With regard to ratings for phenomenal characteristics (MCQ) for past and future events, data were analyzed using 2 (group) by 2 (event valence) ANOVAs. When the interaction was significant, between-group differences for each event valence were examined with $t$ tests, using a Bonferroni correction $(\alpha=0 \cdot 025)$. Finally, for AM measures that significantly differed between alcoholics and controls, the relationships with clinical variables (number of prior detoxification treatments, daily alcohol consumption, number of days of alcohol abstinence, BDI scores, and STAI trait and state scores) were examined with Pearson correlations, using a Bonferroni correction. All tests were two-tailed and were performed with STATISTICA 6.0 (StatSoft Inc., Tulsa, OK, USA).

\section{RESULTS}

\section{AMT}

The mean number of omissions was 2.35 for alcoholics (s.D. $=2.01$ ) and 1.40 for controls $($ S.D. $=1.47)$, but this difference failed to reach statistical significance $[t(38)=1 \cdot 71, p=0 \cdot 10]$. Mean proportions of specific, extended and categoric memories in alcoholics and controls are shown in Fig. 1. The frequency of specific memories was lower in alcoholics than in controls $[t(38)=-3 \cdot 50, p=0 \cdot 001]$. However, both extended and categoric memories were more frequent in alcoholics than in controls $[t(38)=$ $2 \cdot 04, p=0.048$ and $t(38)=2.35, p=0.02$ respectively].

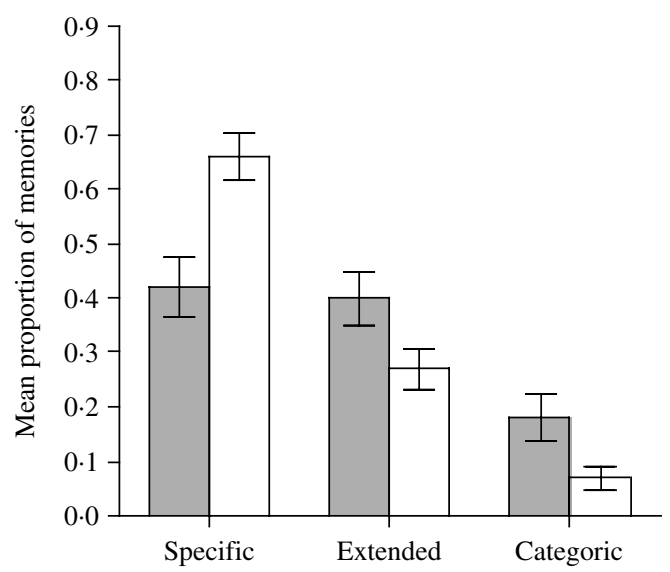

FIG. 1. Mean proportions ( \pm S.E.M.) of specific, extended and categoric memories recalled in the Autobiographical Memory Test. $\square$, Alcoholics $(n=20) ; \square$, controls $(n=20)$.

\section{Ratings for phenomenal characteristics (MCQ)}

Mean ratings for phenomenal characteristics for past and future events are presented in Table 2 as a function of group (alcoholics, controls) and event valence (positive, negative). For past events, there was a main effect of valence for sensory details $[F(1,38)=24 \cdot 71, p<0 \cdot 001]$, and a group $\times$ event valence interaction $[F(1,38)=$ 4.04, $p=0.05$ ). Subsequent $t$ tests (using $\alpha=0.025$, corrected for multiple comparisons) showed a trend towards a significant difference between alcoholics and controls for positive past events $[t(38)=2 \cdot 21, p=0 \cdot 026]$, suggesting that ratings were higher for alcoholics than for controls; there was no significant difference between the two groups for negative past events $[t(38)=0 \cdot 34, p=0 \cdot 73]$. There was no significant main effect of group or interaction involving the group factor for the remaining phenomenal characteristics (all $p$ 's $>0 \cdot 13$ ).

For future events, there was a main effect of group for contextual details $[F(1,38)=6 \cdot 77$, $p=0 \cdot 01]$, with the context of future events being less clearly represented by alcoholics than by controls. The main effect of valence approached significance $[F(1,38)=3 \cdot 16, p=0 \cdot 08]$ but did not interact with group $[F(1,38)=2 \cdot 07, p=0 \cdot 16]$. There was no significant main effect of group or interaction involving the group factor for the remaining phenomenal characteristics (all $p$ 's $>0 \cdot 14)$. 
Table 2. Mean ratings for phenomenal characteristics when remembering past events and imagining future events

\begin{tabular}{|c|c|c|c|c|c|c|c|c|}
\hline & \multicolumn{4}{|c|}{ Alcoholics $(n=20)$} & \multicolumn{4}{|c|}{ Controls $(n=20)$} \\
\hline & \multicolumn{2}{|c|}{ Past events } & \multicolumn{2}{|c|}{ Future events } & \multicolumn{2}{|c|}{ Past events } & \multicolumn{2}{|c|}{ Future events } \\
\hline & Positive & Negative & Positive & Negative & Positive & Negative & Positive & Negative \\
\hline Sensory details & $5 \cdot 67 \pm 1 \cdot 41$ & $4 \cdot 13 \pm 1 \cdot 53$ & $4 \cdot 43 \pm 1 \cdot 79$ & $3 \cdot 40 \pm 2 \cdot 22$ & $4 \cdot 63 \pm 1 \cdot 42$ & $3 \cdot 98 \pm 1 \cdot 22$ & $4 \cdot 50 \pm 1 \cdot 69$ & $2 \cdot 93 \pm 1 \cdot 19$ \\
\hline Contextual information & $6 \cdot 19 \pm 0.87$ & $5 \cdot 61 \pm 1 \cdot 47$ & $3 \cdot 25 \pm 1 \cdot 68$ & $2 \cdot 30 \pm 1 \cdot 95$ & $5.99 \pm 0.73$ & $6 \cdot 06 \pm 0 \cdot 86$ & $4 \cdot 01 \pm 1 \cdot 62$ & $3.91 \pm 1.62$ \\
\hline Other-referential information & $5 \cdot 43 \pm 1 \cdot 73$ & $5 \cdot 05 \pm 2 \cdot 34$ & $5 \cdot 00 \pm 2 \cdot 22$ & $4 \cdot 18 \pm 2 \cdot 42$ & $4 \cdot 83 \pm 1 \cdot 73$ & $5 \cdot 35 \pm 1 \cdot 26$ & $3 \cdot 98 \pm 1 \cdot 71$ & $3 \cdot 40 \pm 1 \cdot 87$ \\
\hline Perspective & $-0.95 \pm 2.56$ & $-0 \cdot 50 \pm 2 \cdot 70$ & $0 \cdot 35 \pm 2 \cdot 39$ & $-0 \cdot 10 \pm 2 \cdot 61$ & $-0 \cdot 85 \pm 2 \cdot 23$ & $-1 \cdot 00 \pm 2 \cdot 22$ & $-0.05 \pm 2.63$ & $0 \cdot 10 \pm 2 \cdot 47$ \\
\hline Positive emotions & $6 \cdot 85 \pm 0.49$ & $1 \cdot 85 \pm 2 \cdot 08$ & $6.45 \pm 1.05$ & $2 \cdot 85 \pm 2 \cdot 58$ & $6 \cdot 15 \pm 1 \cdot 35$ & $1.85 \pm 1.69$ & $6 \cdot 45 \pm 0.76$ & $2 \cdot 10 \pm 1 \cdot 74$ \\
\hline Negative emotions & $1.90 \pm 1.97$ & $6 \cdot 60 \pm 0.88$ & $2 \cdot 45 \pm 2 \cdot 01$ & $5 \cdot 80 \pm 2 \cdot 21$ & $1 \cdot 85 \pm 1 \cdot 81$ & $6 \cdot 45 \pm 0.89$ & $1 \cdot 80 \pm 0 \cdot 83$ & $5 \cdot 75 \pm 1 \cdot 52$ \\
\hline Temporal distance (months) & $119 \pm 104$ & $136 \pm 135$ & $19 \pm 22$ & $43 \pm 131$ & $88 \pm 81$ & $101 \pm 130$ & $26 \pm 18$ & $37 \pm 77$ \\
\hline
\end{tabular}

Values are means \pm s.D.

\section{Correlations with clinical variables}

We examined the relationships between the clinical variables (number of prior detoxification treatments, daily alcohol consumption, number of days of alcohol abstinence, BDI scores, and STAI trait and state scores) and the AM measures that differed significantly between controls and alcoholic patients. With regard to the AMT, no correlation achieved statistical significance (at $p<0 \cdot 0028$, corrected for multiple comparisons); there was only a trend towards significant correlations between BDI scores and proportions of specific $(r=-0 \cdot 41, p=0 \cdot 07)$ and categoric $(r=0.44, p=0.06)$ memories. With regard to ratings for phenomenal characteristics, no correlation achieved statistical significance (at $p<0.0028$, corrected for multiple comparisons).

\section{DISCUSSION}

This study examined AM in non-amnesic alcoholic patients by assessing $(a)$ the frequency with which they retrieve specific versus overly general (categoric and extended) AMs in response to cue words, and $(b)$ their conscious experience when they remember specific past events and when they project themselves into specific future events. The results of the AMT showed that alcoholics reported specific memories less frequently and categoric and extended memories more frequently than healthy controls. To recall a specific memory in the AMT, the rememberer has to elaborate the cue word to search for information in memory, which typically results in the retrieval of a general event, and then to further elaborate the retrieved information and recycle it in another search, until event-specific knowledge is accessed. This complex iterative retrieval process depends crucially on the frontal lobes, which allow one to access event-specific knowledge situated in posterior networks (in the temporal and occipital regions), through abstract knowledge (lifetime periods and general events) situated in frontotemporal anterior regions (Conway \& Pleydell-Pearce, 2000). Indeed, the retrieval of specific AMs can be impaired following damage to the frontal lobes (Piolino et al. 2003; Levine, 2004), and functional neuroimaging studies almost invariably show prefrontal activations during AM retrieval (Gilboa, 2004). Accordingly, it is very likely that the reduced frequency of specific AMs and increased frequency of categoric and extended AMs observed in alcohol-dependent patients result from changes in frontal lobe function that are associated with alcoholism (Moselhy et al. 2001); these changes cause retrieval search operations to terminate more often at the level of general events. Furthermore, difficulties of alcoholic patients in accessing event-specific knowledge may have been aggravated by state-dependent memory effects. There is indeed evidence that information learned under the influence of alcohol is more easily accessed when memory is also tested under the influence of alcohol compared to a placebo (Duka et al. 2001). Assuming that at least some AMs of the alcoholic patients were encoded when they were actively drinking, accessing event-specific knowledge for these AMs may have been more difficult because the 
internal state of the patients changed between encoding and retrieval (i.e. they stopped drinking). Nevertheless, when retrieval difficulties are overcome and a specific past event is accessed, alcoholic patients subjectively experience as many event-specific details (sensory, contextual, self-referential and other-referential) as healthy controls, as indicated by our results concerning MCQ ratings.

While the two groups did not differ in their MCQ ratings for contextual details when they remembered specific past events, alcoholic patients subjectively experienced fewer contextual details than controls when they imagined specific future events. It has been convincingly argued that remembering the past and imagining the future may involve very similar mechanisms (Suddendorf \& Corballis, 1997; Atance \& O'Neill, 2001). As mentioned above, memories for past events are transient mental representations constructed from specific sensoryperceptual details, as well as more abstract knowledge. Similarly, mentally simulating future events involves combining some basic elements (e.g. actors, objects, and actions), some of which are extrapolations from specific past events while others come from abstract knowledge, to generate potential scenarios. Although the mechanisms may be basically the same in the two cases, mentally simulating future events might nevertheless be more resource demanding than remembering past events because of the almost infinite combinations that might be constructed concerning the future and this may be particularly the case for contextual information. Indeed, although one may access sensory information about people and objects that could be involved in a future event relatively easily, the process of constructing a coherent and plausible context that situates these elements in space and time is probably more resource demanding. This might explain why the context of imagined future events was subjectively less detailed in alcoholics than in controls. Another finding of this study was that alcoholics showed a trend towards subjectively experiencing more sensory details than controls when they remembered positive past events. This finding was unexpected and we have no ready explanation for it. Perhaps it reflects an attempt by recently detoxified alcoholics to focus more on their positive past experiences in order to distract themselves from their present difficulties and/or to seek encouragement in going through the detoxification process. Future studies should be conducted to shed some light on this memory enhancement for positive past experiences.

Although patients who met the criteria for a major depressive episode were excluded from our sample, alcoholic patients had more depressive symptoms (as revealed by the BDI) than healthy controls. Williams (1996) argued that overgeneral AM (i.e. difficulties in accessing memories for events that happened at a particular place and time) is an enduring cognitive style for retrieval that underlies vulnerability to clinical depression and is independent of current mood state. Studies showing that the overgenerality pattern of AM remains stable after remission from depression are in agreement with this hypothesis (Brittlebank et al. 1993; Mackinger et al. 2000). However, some studies found that the induction of a depressed mood in non-depressed individuals is associated with an increase in categoric memories and/or a decrease in specific memories (Maccallum et al. 2000; McBride \& Cappeliez, 2004), suggesting that mood may play a significant role. This raises the possibility that depressive symptoms might have contributed to the AM differences between alcoholics and controls in this study. It is worth noting that depressive symptoms are common during alcohol withdrawal (Schuckit et al. 1997; Andersohn \& Kiefer, 2004), so the use of statistical procedures such as analyses of covariance (ANCOVAs) to control for the group difference in depressive symptoms is inappropriate because the covariate (the BDI scores) is not independent of group membership (Miller \& Chapman, 2001).

Although we cannot exclude the possibility that depressive symptoms played a role in the AM differences between the two groups, one aspect of our findings is difficult to explain with reference only to differences in depressed mood. The AM response pattern that had been observed both in depressed patients and in nondepressed individuals in whom a depressed mood was induced consisted in an increase in categoric memories and/or a decrease in specific memories, but there was no increase in extended memories (Williams, 1996; McBride \& Cappeliez, 2004). By contrast, our alcoholdependent group showed an increase in the 
frequency of both categoric and extended memories. Thus, the AM retrieval difficulties that were observed in alcoholic patients were broader than those that would be expected on the basis of depressive symptoms alone. Instead, as suggested above, the frontal lobe deficits that are associated with alcoholism might cause the search process for specific memories to abort prematurely, resulting in more frequent categoric and extended responses. Nevertheless, it must be acknowledged that the occurrence of depressive symptoms in alcoholics may exacerbate some of their AM impairments, as suggested by a trend towards significant correlations between BDI scores and the proportions of specific and categoric memories observed in alcoholic patients.

Finally, it is worth noting that AM is crucial for everyday functioning, at both the intrapersonal (self) and the interpersonal (social) level (Bluck, 2003). Specific AMs carry information about how one dealt with a particular situation (e.g. the outcomes of previous choices and plans), and so they facilitate decision making and plan formulation by enabling intentional choices among alternative courses of action with different goals (Conway, 2001; Pillemer, 2003). Thus, the ability to retrieve specific AMs is believed to play a crucial role in interpersonal problem solving (Goddard et al. 1996; Williams, 1996). In addition, specific AMs plays an important role in developing, maintaining and strengthening social bonds by providing material for conversations (Bluck, 2003). Therefore, the reduced ability of alcoholdependent patients to retrieve specific memories might place them in a less comfortable position when dealing with current challenges in their social environment, and this might be a risk factor for having a relapse.

\section{ACKNOWLEDGMENTS}

We thank Romaine Birden for her help in recruiting and testing participants. Arnaud D'Argembeau is a Postdoctoral Researcher at the Belgian National Fund for Scientific Research (FNRS).

\section{DECLARATION OF INTEREST}

None.

\section{REFERENCES}

Andersohn, F. \& Kiefer, F. (2004). Depressive mood and craving during alcohol withdrawal: association and interaction. German Journal of Psychiatry 7, 6-11.

Atance, C. M. \& O’Neill, D. K. (2001). Episodic future thinking. Trends in Cognitive Sciences 5, 533-539.

Beck, A., Rial, W. \& Rickets, K. (1974). Short form of depression inventory: cross-validation. Psychological Report 34, 1184-1186.

Bluck, S. (2003). Autobiographical memory: exploring its function in everyday life. Memory 11, 113-123.

Brandt, J., Butters, N., Ryan, C. \& Bayog, R. (1983). Cognitive loss and recovery in long-term alcohol abusers. Archives of General Psychiatry 40, 435-442.

Brittlebank, A. D., Scott, J., Williams, J. M. G. \& Ferrier, I. N. (1993). Autobiographical memory in depression: state or trait marker? British Journal of Psychiatry 162, 118-121.

Conway, M. A. (2001). Sensory-perceptual episodic memory and its context: autobiographical memory. Philosophical Transactions of the Royal Society of London 356, 1375-1384.

Conway, M. A. \& Pleydell-Pearce, C. W. (2000). The construction of autobiographical memories in the self-memory system. Psychological Review 107, 261-288.

D'Argembeau, A. \& Van der Linden, M. (2004). Phenomenal characteristics associated with projecting oneself back into the past and forward into the future: influence of valence and temporal distance. Consciousness and Cognition 13, 844-858.

Duka, T., Weissenborn, R. \& Dienes, Z. (2001). State-dependent effects of alcohol on recollective experience, familiarity and awareness of memories. Psychopharmacology 153, 295-306.

Fitzgerald, J. M. \& Shifley-Grove, S. (1999). Memory and affect: autobiographical memory distribution and availability in normal adults and recently detoxified alcoholics. Journal of Adult Development 6, 11-19.

Gilboa, A. (2004). Autobiographical and episodic memory - one and the same? Evidence from prefrontal activation in neuroimaging studies. Neuropsychologia 42, 1336-1349.

Goddard, L., Dritschel, B. \& Burton, A. (1996). Role of autobiographical memory in social problem solving and depression. Journal of Abnormal Psychology 105, 609-616.

Grant, I. (1987). Alcohol and the brain: neuropsychological correlates. Journal of Consulting and Clinical Psychology 55, 310-324.

Heffernan, T. M., Moss, M. \& Ling, J. (2002). Subjective ratings of prospective memory deficits in chronic heavy alcohol users. Alcohol and Alcoholism 37, 269-271.

Johnson, M. K., Foley, M. A., Suengas, A. G. \& Raye, C. L. (1988) Phenomenal characteristics of memories for perceived and imagined autobiographical events. Journal of Experimental Psychology: General 117, 371-376.

Kopelman, M. D. \& Kapur, N. (2001). The loss of episodic memories in retrograde amnesia: single-case and group studies. Philosophical Transactions of the Royal Society of London 356, 1409-1421.

Levine, B. (2004). Autobiographical memory and the self in time: brain lesion effects, functional neuroanatomy, and lifespan development. Brain and Cognition 55, 54-68.

Ling, J., Heffernan, T. M., Buchanan, T., Rodgers, J., Scholey, A. B. \& Parrott, A. C. (2003). Effects of alcohol on subjective ratings of prospective and everyday memory deficits. Alcoholism: Clinical and Experimental Research 27, 970-974.

Maccallum, F., McConkey, K. M., Bryant, R. A. \& Barnier, A. J. (2000). Specific autobiographical memory following hypnotically induced mood state. International Journal of Clinical and Experimental Hypnosis 48, 361-373.

Mackinger, H. F., Pachinger, M. M., Leibetseder, M. M. \& Fartacek, R. R. (2000). Autobiographical memories in women remitted from major depression. Journal of Abnormal Psychology 109, 331-334.

McBride, C. \& Cappeliez, P. (2004). Effects of manipulating valence and arousal components of mood on specificity of autobiographical memory. Psychological Reports 95, 615-630.

McNally, R. J., Lasko, N. B., Macklin, M. L. \& Pitman, R. K. (1995). Autobiographical memory disturbance in combat-related 
posttraumatic stress disorder. Behaviour Research and Therapy 33, 619-630.

Miller, G. A. \& Chapman, J. P. (2001). Misunderstanding analysis of covariance. Journal of Abnormal Psychology 110, 40-48.

Moselhy, H. F., Georgiou, G. \& Kahn, A. (2001). Frontal lobe changes in alcoholism: a review of the literature. Alcohol and Alcoholism 36, 357-368.

Nigro, G. \& Neisser, U. (1983). Point of view in personal memories. Cognitive Psychology 15, 467-482.

Noël, X., Van der Linden, M., Schmidt, N., Sferrazza, R., Hanak, C., Le Bon, O., De Mol, J., Kornreich, C., Pelc, I. \& Verbanck, P. (2001). Supervisory attentional system in nonamnesic alcoholic men. Archives of General Psychiatry 58, 1152-1158.

Parsons, O. A. (1998). Neurocognitive deficits in alcoholics and social drinkers: a continuum? Alcoholism: Clinical and Experimental Research 22, 954-961.

Pillemer, D. B. (2003). Directive functions of autobiographical memory: the guiding power of the specific episode. Memory 11, 193-202.

Piolino, P., Desgranges, B., Belliard, S., Matuszewski, V., Lalevee, C., De la Sayette, V. \& Eustache, F. (2003). Autobiographical memory and autonoetic consciousness: triple dissociation in neurodegenerative diseases. Brain 126, 2203-2219.

Riutort, M., Cuervo, C., Danion, J. M., Peretti, C. S. \& Salame, P. (2003). Reduced levels of specific autobiographical memories in schizophrenia. Psychiatry Research 117, 35-45.

Rubin, D. C., Schrauf, R. W. \& Greenberg, D. L. (2003). Belief and recollection of autobiographical memories. Memory and Cognition 31, 887-901.

Scheurich, A. (2005). Neuropsychological functioning and alcohol dependence. Current Opinion in Psychiatry 18, 319-323.
Schuckit, M. A., Tipp, J. E., Bergman, M., Reich, W., Hesselbrock, V. M. \& Smith, T. L. (1997). Comparison of induced and independent major depressive disorders in 2,945 alcoholics. American Journal Psychiatry 154, 948-957.

Schwartz, B. L., Parker, E. S., Deutsch, S. I., Rosse, R. B., Kaushik, M. \& Isaac, A. (2002). Source monitoring in alcoholism. Journal of Clinical and Experimental Neuropsychology 24, 806-817.

Spielberger, C. D. (1993). Inventaire d'Anxiété Etat-Trait (Forme Y). Les Editions du Centre de Psychologie Appliquée: Paris.

Suddendorf, T. \& Corballis, M. C. (1997). Mental time travel and the evolution of the human mind. Genetic, Social, and General Psychology Monographs 123, 133-167.

Sullivan, E. V., Rosenbloom, M. J. \& Pfefferbaum, A. (2000). Pattern of motor and cognitive deficits in detoxified alcoholic men. Alcoholism: Clinical and Experimental Research 24, 611-621.

Van Vreeswijk, M. F. \& De Wilde, E. J. (2004). Autobiographical memory specificity, psychopathology, depressed mood and the use of the Autobiographical Memory Test: a meta-analysis. Behaviour Research and Therapy 42, 731-743.

Williams, J. M. G. (1996). Depression and the specificity of autobiographical memory. In Remembering our Past: Studies in Autobiographical Memory (ed. D. C. Rubin), pp. 244-267. Cambridge University Press: Cambridge.

Williams, J. M. G. \& Broadbent, K. (1986). Autobiographical memory in suicide attempters. Journal of Abnormal Psychology 95, 144-149.

Williams, J. M. G., Teasdale, J. D., Segal, Z. V. \& Soulsby, J. (2000). Mindfulness-based cognitive therapy reduces overgeneral autobiographical memory in formerly depressed patients. Journal of Abnormal Psychology 109, 150-155. 